\title{
What precedes development of rheumatoid arthritis?
}

\section{Klareskog, L Alfredsson, S Rantapää-Dahlqvist, E Berglin, P Stolt, L Padyukov}

Ann Rheum Dis 2004;63(Suppl II):ii28-ii31. doi: 10.1136/ard.2004.028225

\begin{abstract}
Studies on aetiology of inflammatory diseases such as rheumatoid arthritis (RA) need to investigate the potential environmental triggers that are active before onset of disease, the genetic context in which these triggers act, and whether the presence of such triggers in an arthritis prone genetic context will give rise to the immune reactions associated with/preceding RA. Such knowledge would help not only to address much better the issue of causality of these potential triggers and the immune reactions, but also to carry out various interventions aimed at influencing the disease provoking immune events before development of clinical signs of disease.

This short report summarises recent data demonstrating (a) the presence of anticitrullin antibodies or rheumatoid factors in between a third and half of patients with RA before development of clinical signs; (b) long term smoking is associated with a high risk of future development of seropositive but not seronegative RA; and (c) a strong gene-environment interaction between smoking and SE genes in the development of seropositive RA.

We conclude that, in a certain genetic context, smoking is a potential trigger of RA, and a combination of the two factors is associated with the occurrence of immune reactions long before the onset of RA.
\end{abstract}

$\mathrm{R}$ heumatoid arthritis (RA) is classically described as an "autoimmune disease of unknown aetiology", where genes and the environment contribute to the development of disease. ${ }^{1}$ The obvious questions to ask are:

- Which immune reactions are associated with the disease, and which immune reactions may actually cause the disease?

- What triggers are involved?

- In which genetic context are potential triggers of the disease associated with later emergence of the disease?

- In which order do all these events take place?

- Are there differences in these critical aspects-triggers, genes, and immune events - in individuals with different subtypes of the disease?

RA is a disease defined by clinical criteria. Thus we can expect different pathogenetic pathways to result in clinical conditions with enough similarities to be classified into the entity we currently call RA.

The aim of this report is to discuss and summarise some recent data which may contribute to the formation of a more coherent hypothesis of RA that takes into account triggers, genes, and autoimmune reactions occurring in the years preceding the actual onset of the disease. Figure 1 gives a longitudinal view of the disease in RA where we need to study events occurring before the onset of the first symptoms of undifferentiated arthritis towards the eventual fulfilment of the criteria for RA and development of different pathological consequences of the disease, including comorbidities such a cardiovascular disease and lymphomas. At each step, it may be assumed that different triggers and different genetic constituents are acting along with different immune reactions. Of interest is the fact that a large part of our understanding of experimental arthritis in rodents (see lower part of fig 1) has come from studies of events that occur before the onset of disease. Not least interesting is that interventions which modify or stop future development of disease in these experimental models are most efficient during this period.

\section{IMMUNE REACTIONS OCCURRING BEFORE ONSET OF RA}

It can be hypothesised that specific immune reactions that actually cause disease development in RA should be possible to detect before onset of the disease; else it is difficult to argue for a cause-effect relationship. For a long time only a few studies addressed this question, and the main data came from the Finnish studies by Aho and collaborators, who, having identified pre-RA sera in large Finnish population based biobanks, demonstrated the presence of rheumatoid factors (RFs) many years before onset of seropositive RA. ${ }^{2}$

Recently, this approach was adopted and developed in studies using two different historical biobanks in Sweden and the Netherlands. In these studies it was possible to investigate not only RF but also other recently recognised and more RA specific antibodies, namely antibodies towards citrullinated peptides. ${ }^{3-7}$ In the first study, using a biobank of approximately 90000 individuals from northern Sweden, Rantapää-Dahlqvist and collaborators were able to demonstrate the presence of anticitrullin antibodies as well as RFs up to 10 years before onset of disease. Their approach consisted of a nested case-control study where patients with recent onset of RA were identified from among individuals who had previously given blood samples to the biobank, and the frequency of anticitrullin antibodies in individuals who later developed RA was compared with matched controls. They found that $34 \%$ of the "pre-RA individuals" (individuals who later developed RA) had anticitrullin antibodies compared with less than $2 \%$ of matched population controls.

Using a similar approach, a Dutch research group took advantage of an existing large biobank for individuals who had been blood donors in the Amsterdam region since the 1980s. ${ }^{4}$ They found increased levels of anticitrullin antibodies and RFs in pre-RA sera, which were very similar to those found in the Swedish study-that is, approximately $24 \%$ of individuals who later developed RA had developed anticitrullin antibodies before onset of disease compared with less than $1 \%$ of controls (who were on average younger than the Swedish control cohort).

Abbreviations: RA, rheumatoid arthritis, RF, rheumatoid factor; SE, shared epitope 

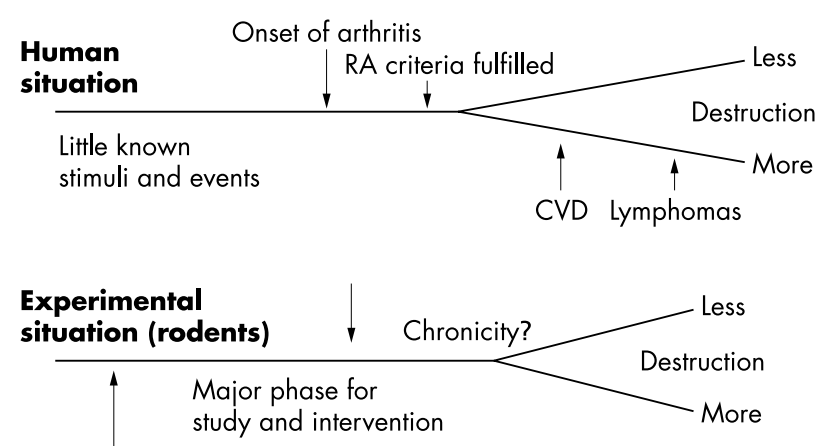

Immunisations etc

(defined stimuli)

Figure 1 Schematic comparison of the events occurring in the development and course of rheumatoid arthritis (RA) in humans and experimentally induced arthritis in rodents.

\section{PREDICTION OF RA: COMBINATION OF GENETIC SUSCEPTIBILITY AND PRESENCE OF ANTICITRULLIN ANTIBODIES GIVES A HIGH RISK FOR FUTURE DEVELOPMENT OF RA}

On the basis of the observation that anticitrullin antibodies are present in high fractions in pre-RA sera, and the well known association between certain alleles of human leucocyte antigen (HLA)-DR (carrying the so called "shared epitope" (SE) $),{ }^{8-10}$ we hypothesised that a combined analysis of pre-RA serology and genotype for SE would predict the risk for RA with even greater precision than either of these factors alone. We carried out a study with the help of the previously mentioned northern Sweden biobank, and the odds ratio for future development of RA in individuals who both carry the SE and are positive for anticitrullin antibodies was found to be as high as $66 .{ }^{11}$

The existence of high predictability using only two factors has a number of obvious implications for research, clinical practice, and ethical handling of information that now need to be addressed in many different fora. In this short report we will restrict ourselves to a part of the future research agenda, namely how this information may be used as a starting point for aetiological studies, taking into account that we now need to consider events triggering immune reactions over at least a 10 year period preceding clinical symptoms of RA.

\section{GENES AND THE ENVIRONMENT IN THE DEVELOPMENT OF RA}

The data reviewed in the previous section highlight the need to study putative environmental stimuli that may act over many years, as well as the genetic contexts that may permit these agents to exert proarthritogenic effects. When conducting such research, the different forms of RA need to be studied separately. Little effort has been made previously to study putative interactions between environmental risk factors and susceptibility genes in RA. One obvious reason for this scarcity of studies is the relative lack of knowledge concerning environmental risk factors for RA. So far, smoking is the only factor that has been unambiguously demonstrated in several studies. ${ }^{12-16}$ Interestingly, in the context of our present reasoning of long term exposure before disease onset, we recently demonstrated that smoking over a long period of time- - that is, at least 20 years, was needed to significantly increase the risk for RA, and, similarly, cessation of smoking reduced the risk only after $10-15$ years. ${ }^{16}$ Taken together, these data indicate that smoking may exert proarthritogenic effects through repeated stimulation over a period of several years.
To study gene-environment interactions in RA, there are in essence two possible methodological approaches. The first is the cohort approach, where a large group of individuals is followed over many years, and all individuals within that group have similar characteristics in a number of respects, such as exposure to different disease risk factors (such as smoking), genotype, and development of the disease of interest. This type of study is optimal from an epidemiological perspective, but the numbers of individuals as well as the numbers of exposures and outcomes that need to be included makes this type of study very difficult in practice. Consequently, no study on gene-environment interactions in RA using this approach has so far been reported.

The other approach is the case-control study, for which it is easier to get access to the appropriate numbers of cases and controls required for analysis, but where strict criteria should be set for unbiased selection of cases and controls and for the future unbiased surveillance and collection of data from the cases and controls. So far, only one small study using this approach has been published, which showed a modest interaction between smoking and an allelic variant of glutathione reductase. ${ }^{17}$

A

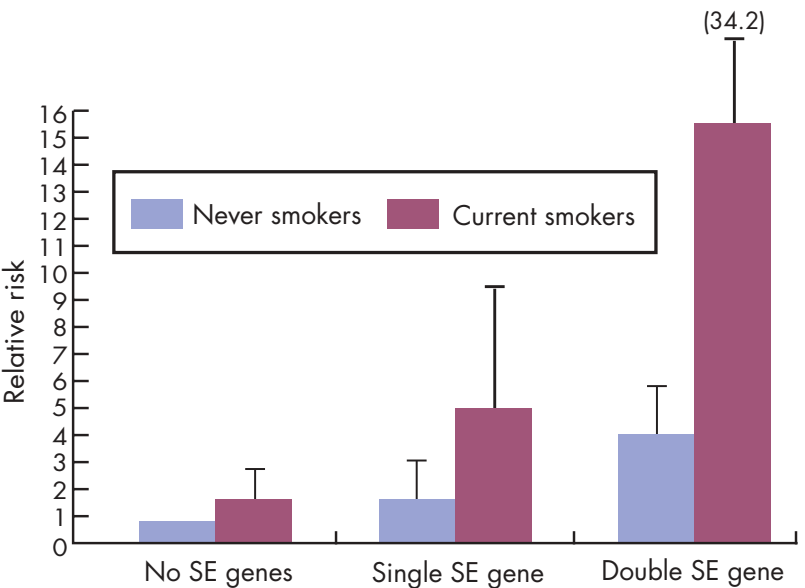

B

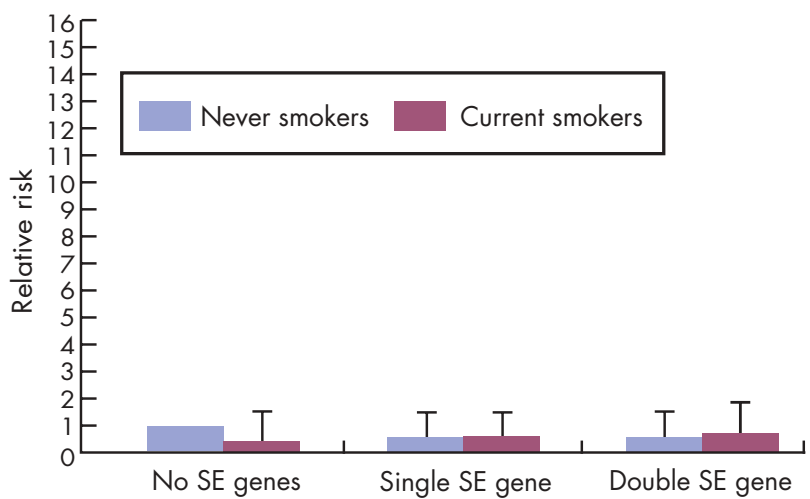

Figure 2 Relative risk (RR) for development of rheumatoid arthritis (RA) in current smokers (with different numbers of copies (0-2) of the shared epitope (SE) of HLA-DR) compared with never smokers. (A) RR for seropositive RA and (B) RR for seronegative RA. These graphs are schematic representations of the original data from a case-control study of RA reported in reference 9 . 


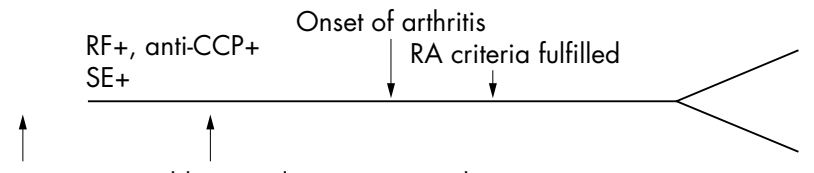

It is now possible to study environmental stimuli, immune events, and interventions in these phases of development of RA

Figure 3 Schematic outline of how aetiological studies as well as interventions in the pathways leading to rheumatoid arthritis (RA) should be undertaken before onset of clinical signs of RA. CCP, cyclic citrullinated peptide; RF, rheumatoid factor; SE shared epitope.

We have, over a period of several years, been building up a large database in a case-control study for analysing geneenvironment interactions in RA with the aim of achieving numbers sufficient to permit appropriate analyses and address the various pitfalls (that could occur) with regard to biased recruitment as well as biased ascertainment of risk factors. Our approach has involved taking advantage of an existing Swedish national initiative for surveillance of all patients with early RA-that is, we asked the patients included in this study (and their doctors) to participate in a study of aetiology of RA. After obtaining informed consent the patietns were asked for detailed information by means of a questionnaire on their lifestyle and environmental exposures (including a detailed history of smoking), and they were also asked to give blood samples for serology and genotyping. An identical procedure was undertaken for controls randomly selected from the population registry and matched with the cases for sex, age, and area of residence.

Currently, this case-control study includes information from 1800 cases and 1800 controls; the data of approximately 1000 cases and an equal number of controls have been subjected to a preliminary analysis of environmental and genetic factors predisposing for arthritis. In this analysis, we first concentrated on the most obvious factors-that is, the contribution of the major genetic factor, the SE genes of HLADR-, and the major known environmental factor, smoking. We also subdivided the patients into the two classic subphenotypes RF+ and RF- (RF typing done at the onset of the disease). The results of this initial exercise are shown schematically in fig 2 (further details are available in the original paper ${ }^{18}$ ). As seen in fig $2 \mathrm{~A}$, both SE and smoking are risk factors for RF+ RA with marked interaction between the environmental exposure and the genetic risk factor (also evident by formal testing of the interaction ${ }^{19}$ ), indicating that individuals carrying double copies of the SE genes and who are smokers are at approximately 16 times increased risk for RF+ RA compared with individuals who neither smoke nor carry any copy of the SE genes. Figure 2B clearly demonstrates that the two risk factors neither alone nor in combination provide any risk for development of seronegative RA.

Three major conclusions may be drawn from these observations: first, a major risk factor for RA, the presence of SE genes, is highly dependent on the frequency of smokers in the population at risk; secondly, genetic as well as environmental risk factors for seropositive and seronegative RA may differ fundamentally; thirdly, smoking over many years, and in the presence of the SE genes, may exert effects on the immune system that make some individuals susceptible to development of RA.

The last observation obviously provides the basis for additional studies to find out whether smoking in the context of SE genes (and potentially other genes as well) may trigger production of not only $\mathrm{RF}^{2}$ but also other antibodies such as anticitrullin antibodies. We are presently addressing this possibility in our case-control study and unpublished results indicate that anticitrullin antibodies may indeed be triggered by smoking in the context of SE genes.

\section{CONCLUDING REMARKS}

This short summary of some recent work on genes, the environment, and specific immune reactions in RA indicates that we are in a better situation now than a few years ago with regard to a number of aetiological issues in RA: we know of at least one potential trigger (long term smoking) which in a specific genetic context (SE of HLA-DR) appears to be able to trigger development of RF (and possibly anticitrullin antibodies), with both the RFs and the anticitrullin antibodies appearing before onset of RA. Emerging knowledge of these critical events puts us in a situation (fig 3) where we are now able to further analyse environmental, genetic, and immune events which drive or counteract development of RA, and potentially also intervene with these events even before the onset of disease.

\section{ACKNOWLEDGEMENTS}

The case-control study on rheumatoid arthritis in Sweden referred to in the report would not have been possible without the contributions from the many rheumatology units participating in the EIRA study (for a complete list of acknowledgements, see reference 16).

\section{Authors' affiliations}

L Klareskog, L Padyukov, Rheumatology Unit, Department of Medicine, Karolinska Institutet/Karolinska University Hospital, Stockholm, Sweden L Alfredsson, P Stolt, Institute for Environmental Medicine, Karolinska Institutet, Stockholm, Sweden

S Rantapää-Dahlqvist, E Berglin, Department of Rheumatology, Umeå University and University Hospital, Umeå, Sweden

The studies from our own laboratory referred to in this report were supported by grants from the Swedish Research Council, the insurance company AFA, King Gustaf Vth 80-year Foundation and the Swedish Association against Rheumatism.

During the preparation of this article, LK received support from the Fernström Foundation to spend time as guest scientist at Harvard Medical School (Department of Rheumatology and Department of Neuroscience).

Correspondence to: L Klareskog, Rheumatology Unit, Department of Medicine, Karolinska University Hospital, 17176 Stockholm, Sweden; Lars.Klareskog@medks.ki.se

\section{REFERENCES}

1 Klareskog L, Lorentzen J, Padyukov L, Alfredsson L. Genes and environment in arthritis: can RA be prevented? Arthritis Res 2002;4(suppl 3):31-6.

2 Aho K, Heliovaara M, Maatela J, Tuomi T, Palosuo T. Rheumatoid factor antedating clinical rheumatoid arthritis. J Rheumatol 1991;18:1282-4.

3 Rantapää-Dahlqvist S, de Jong BA, Berglin E, Hallmans G, Wadell G, Stenlund $\mathrm{H}$, et al. Antibodies against cyclic citrullinated peptide and $\lg \mathrm{A}$ rheumatoid factor predict the development of rheumatoid arthritis. Arthritis Rheum 2003;48:2741-9.

4 Nielen MM, van Schaardenburg D, Reesink HW, van de Stadt RJ, van der Horst-Bruinsma IE, de Koning MH, et al. Specific autoantibodies precede the symptoms of rheumatoid arthritis: a study of serial measurements in blood donors. Arthritis Rheum, 2004;50, 380-6.

5 van Venrooij W, Pruijn GJM. Citrullination: a small change for a protein with great consequences for rheumatoid arthritis. Arthritis Res 2000;2:249-51.

6 Vossenaar ER, Nijenhuis S, Helsen MM, van der Heijden A, Senshu T, van den Berg WB, et al. Citrullination of synovial proteins in murine models of rheumatoid arthritis. Arthritis Rheum 2003;48:2489-500.

7 Vossenaar ER, Van Venrooij WJ. Citrullinated proteins: sparks that may ignite the fire in rheumatoid arthritis. Arthritis Res Ther 2004;6:107-11.

8 Gregersen PK, Silver J, Winchester RJ. The shared epitope hypothesis. An approach to understanding the molecular genetics of susceptibility to rheumatoid arthritis. Arthritis Rheum 1987;30:1205-13.

9 Gregersen PK. Genetics of rheumatoid arthritis: confronting complexity Arthritis Res 1999;1:37-44.

10 Jawaheer D. Gregersen PK. Rheumatoid arthritis. The genetic components. Rheum Dis North Clin North Am 2002;28:1-15.

11 Berglin E, Padyukov L, Sundin U, Hallmans G, Stenlund H, van Venrooii WJ, et al. A combination of autoantibodies to cyclic citrullinated peptide (CCP) and 
HLA-DRB1 locus antigens is strongly associated with future onset of rheumatoid arthritis. Arthritis Res Ther 2004;6:R303-8, Epub 11 May 2004

12 Vessey MP, Villard-Mackintosh L, Yeates D. Oral contraceptives, cigarette smoking and other factors in relation to arthritis. Contraception 1987;35:457-64.

13 Silman AJ, Newman J, MacGregor AJ. Cigarette smoking increases the risk of rheumatoid arthritis. Results from a nationwide study of disease-discordant twins. Arthritis Rheum 1996;39:732-5.

14 Heliövaara M, Aho K, Aromaa A, Knekt P, Reunanen A. Smoking and risk of rheumatoid arthritis. J Rheumatol 1993;20:1830-5.

15 Uhlig T, Hagen KB, Kvien TK. Current tobacco smoking, formal education, and the risk of rheumatoid arthritis. J Rheumatol 1999;26:47-54.
16 Stolt P, Bengtsson C, Nordmark B, Lindblad S, Lundberg I, Klareskog L, Alfredsson $L$. Quantification of the influence of cigarette smoking on rheumatoid arthritis. Ann Rheum Dis 2003;62:835-41.

17 Mattey DL, Hutchinson D, Dawes PT, Nixon NB, Clarke S, Fisher J, et al. Smoking and disease severity in rheumatoid arthritis: association with polymorphism at the glutathione S-transferase M1 locus. Arthritis Rheum 2002;46:640-6.

18 Padyukov L, Silva C, Stolt P, Alfredsson L, Klareskog L, EIRA study group. Interaction between smoking and shared epitope genes in HLA-DR provides a high risk for seropositive rheumatoid arthritis. Arthritis Rheum 2004 (in press).

19 Rothman KJ, Greenland S, Walker AM. Concepts of interaction. Am J Epidemiol 1980;112:467-70. 